\title{
EXTREME POSITIVE TERNARY SEXTICS
}

\author{
AARON KUNERT, CLAUS SCHEIDERER
}

\begin{abstract}
We study nonnegative (psd) real sextic forms $q\left(x_{0}, x_{1}, x_{2}\right)$ that are not sums of squares (sos). Such a form has at most ten real zeros. We give a complete and explicit characterization of all sets $S \subseteq \mathbb{P}^{2}(\mathbb{R})$ with $|S|=9$ for which there is a psd non-sos sextic vanishing in $S$. Roughly, on every plane cubic $X$ with only real nodes there is a certain natural divisor class $\tau_{X}$ of degree 9 , and $S$ is the real zero set of some psd non-sos sextic if, and only if, there is a unique cubic $X$ through $S$ and $S$ represents the class $\tau_{X}$ on $X$. If this is the case, there is a unique extreme ray $\mathbb{R}_{+} q_{S}$ of psd non-sos sextics through $S$, and we show how to find $q_{S}$ explicitly. The sextic $q_{S}$ has a tenth real zero which for generic $S$ does not lie in $S$, but which may degenerate into a higher singularity contained in $S$. We also show that for any eight points in $\mathbb{P}^{2}(\mathbb{R})$ in general position there exists a psd sextic that is not a sum of squares and vanishes in the given points.
\end{abstract}

\section{INTRODUCTION}

In a famous and influential paper, Hilbert [6] proved in 1888 that a polynomial with real coefficients that takes nonnegative values only can usually not be written as a sum of squares of real polynomials. More precisely, there exist nonnegative ternary forms of any even degree $\geq 6$ that are not sums of squares. For forms in four or more variables, the same is true for even degrees $\geq 4$. Hilbert could well have used his own arguments to construct concrete examples of such forms. However he didn't do so, and it took almost 80 more years before first explicit examples appeared in print.

It has become common use to say that a real form is $p s d$ (positive semidefinite) if it has nonnegative values, and that it is sos (sum of squares) if it is a sum of squares of forms. First examples of psd forms that are not sos were found in the 1960s by Motzkin, Ellison and Robinson, being verified in ad hoc ways. In the 1970s, Choi, Lam and later Reznick started to explore the phenomenon in more systematic ways. In particular, they studied extreme psd forms, which are psd forms that are not squares and cannot be written as a sum of psd forms in a nontrivial way. Closely related is the study of the possible real zero sets of psd non-sos forms. On the other hand they constructed a variety of new examples. See Reznick's paper [8] for a detailed account with precise references of work done up to around the year 2000 . In 9, Reznick formalized the arguments from Hilbert's proof, thereby showing in the case of ternary sextics that whenever two plane cubics intersect in nine different $\mathbb{R}$-points, there exists a psd non-sos sextic through any eight of them.

In this paper we focus entirely on ternary sextics. A psd sextic that is not sos has at most ten real zeros. Our main contribution is a complete and explicit characterization of all sets of nine points in $\mathbb{P}^{2}(\mathbb{R})$ on which some psd non-sos sextic

2010 Mathematics Subject Classification. Primary 14 P 05; secondary 14 C 22, 14 H 45. 
vanishes. To describe the result let $X$ be a reduced plane cubic, and let $S$ be a set of 9 nonsingular $\mathbb{R}$-points on $X$. We say that $S$ is admissible if the class of the Weil divisor $\sum_{P \in S} P$ on $X$ is a non-trivial half of $\left[\mathcal{O}_{X}(6)\right]$, and if this class satisfies a certain definiteness condition (2.9). For every admissible $S$ there exists a psd non-sos sextic that vanishes in $S$. In fact, up to positive scaling there is a unique such sextic, denoted $q_{S}$, that is extreme. Conversely, for every psd non-sos sextic with a set $S$ of nine real zeros, there is a unique cubic $X$ through $S$, and $S$ is admissible in the sense before (4.7).

As for the cubics that arise in this construction, we show that a cubic $X$ contains an admissible set if and only if $X$ has at most real nodes (with real tangents) as singularities. Moreover, the divisor class in question is unique in this case (2.8).

Our characterization is explicit enough to make it easy, for any given set $S$, to check effectively whether $S$ is admissible, and in the positive case, to write down concretely a psd non-sos sextic through $S$, or even the extreme sextic $q_{S}$. In this way we obtain an explicit parametrization of the extreme psd sextics with at least nine real zeros. Note that these sextics are dense inside the set of all extreme psd sextics. Since generically an extreme psd sextic has ten real zeros, our parametrization happens in a 10:1 fashion. Recently, Blekherman et al. showed [1] that the extreme psd sextics form a Zariski dense subset of the Severi variety of all rational sextics. In particular, they form a family of (projective) dimension 17. Our approach gives a new and very clear approach to these facts. We apply our result to prove that any 8 points of the plane are the real zero set of a psd non-sos sextic, unless 4 of them are on a line or 7 are on a conic (4.13). Before, this was known only under additional assumptions.

The paper is organized as follows. In Section 2 we discuss Picard groups of real (possibly singular) curves. We define definite 2-torsion classes in the Picard group and determine these classes in the case of plane cubics. In Section 3 we show that every set of nine real zeros of a psd non-sos sextic is admissible. The converse is proved in Section 4, where we also state a number of complements to the main result. Section 5 contains a series of examples illustrating various aspects of our construction.

\section{Preliminaries and notation}

We work with ternary forms only. Let $d \geq 0$. By $P_{2 d}$ (resp. $\Sigma_{2 d}$ ) we denote the set of all psd (positive semidefinite) forms $f \in \mathbb{R}\left[x_{0}, x_{1}, x_{2}\right]$ with $\operatorname{deg}(f)=2 d$ (resp. its subset of all sos forms, i.e. forms that can be written as a finite sum of squares of forms). It is well known that $\Sigma_{2 d} \subseteq P_{2 d}$ are closed convex cones, and that the inclusion is proper if and only if $2 d \geq 6$.

If $C$ is any convex cone with $C \cap(-C)=\{0\}$, the extreme rays of $C$ are the one-dimensional faces of $C$. In the case of the psd cone $P_{2 d}$ we reserve the term for forms that are not sums of squares. Thus, we will say that a psd form $q \in P_{2 d}$ is extreme if $q \notin \Sigma_{2 d}$, and if $q=q_{1}+q_{2}$ with $q_{1}, q_{2} \in P_{2 d}$ implies $q_{1}, q_{2} \in \mathbb{R}_{+} q$.

All varieties $X$ (usually curves) are defined over $\mathbb{R}$ and are considered as $\mathbb{R}$ schemes. As usual, $X_{\text {sing }}$ resp. $X_{\text {reg }}$ denotes the singular resp. the nonsingular locus of $X$, and $X(\mathbb{R})$ (resp. $X(\mathbb{C})$ ) is the set of $\mathbb{R}$-rational (resp. $\mathbb{C}$-rational) points of $X$. For a form $f \in \mathbb{R}\left[x_{0}, x_{1}, x_{2}\right], V(f)$ is the curve $f=0$ in $\mathbb{P}^{2}$, and $V_{\mathbb{R}}(f)$ denotes the set of its real points. When $f, g \in \mathbb{R}\left[x_{0}, x_{1}, x_{2}\right]$ are forms without 
common irreducible component and $P \in \mathbb{P}^{2}(\mathbb{R})$, the local intersection number of $f$ and $g$ at $P$ is written $i_{P}(f, g)$.

Let $X$ be a curve over $\mathbb{R}$. A scheme point $x \in X$ is called a node (resp. an acnode) of $X$ if $x$ has residue field $\mathbb{R}$ and has multiplicity 2 with two different tangent directions, where the tangents are real resp. complex conjugate. Thus $x$ is a node iff $\widehat{\mathcal{O}}_{X, x} \cong \mathbb{R}[[u, v]] /(u v)$, and is an acnode iff $\widehat{\mathcal{O}}_{X, x} \cong \mathbb{R}[[u, v]] /\left(u^{2}+v^{2}\right)$. The real curve $X(\mathbb{R})$ has two branches intersecting transversally at $x$ when $x$ is a node, and has an isolated point at $x$ when $x$ is an acnode.

For $S \subseteq \mathbb{P}^{2}(\mathbb{R})$ a finite set, we denote by $I_{d}(m S)$ the space of degree $d$ forms in $\mathbb{R}\left[x_{0}, x_{1}, x_{2}\right]$ that have multiplicity $\geq m$ at every point $P \in S$.

\section{PicARD GRoups of REAL CURVES}

We need to work with groups of divisor classes not only on nonsingular (plane) curves, but also on singular and even reducible curves. Therefore we need to discuss Picard groups in this generality.

2.1. Let $X$ be a reduced projective curve over $\mathbb{R}$, always considered as a scheme over $\mathbb{R}$. We allow $X$ to be reducible. By $X_{\mathbb{C}}$ we denote the base field extension $X_{\mathbb{C}}=X \times_{\operatorname{Spec}(\mathbb{R})} \operatorname{Spec}(\mathbb{C})$. The ring of rational functions on $X$ is $\mathbb{R}(X)=$ $H^{0}\left(X, \mathcal{K}_{X}\right)$, and similarly $\mathbb{C}(X)=H^{0}\left(X_{\mathbb{C}}, \mathcal{K}_{X_{\mathbb{C}}}\right)$. The group of Cartier divisors on $X$ is $\operatorname{Div}(X)=H^{0}\left(X, \mathcal{K}_{X}^{*} / \mathcal{O}_{X}^{*}\right)$, linear equivalence of divisors on $X$ is denoted by $\sim$. The Picard group $\operatorname{Pic}(X)$ is (isomorphic to) the group of divisors on $X$ modulo linear equivalence, i.e., the natural sequence

$$
1 \rightarrow \mathcal{O}(X)^{*} \rightarrow \mathbb{R}(X)^{*} \rightarrow \operatorname{Div}(X) \rightarrow \operatorname{Pic}(X) \rightarrow 0
$$

is exact.

For $U \subseteq X$ open there is a natural map $\operatorname{Div}(U) \rightarrow \operatorname{Div}(X)$, the extension by zero. If $U$ is dense in $X$ then this map is surjective up to linear equivalence. In particular, this applies to $U=X_{\text {reg, }}$, the nonsingular locus of $X$. Since Cartier divisors on $X_{\text {reg }}$ can be identified with Weil divisors (zero cycles) on $X_{\text {reg }}$, we will often tacitly represent divisor classes on $X$ by Weil divisors on $X_{\text {reg }}$.

2.2. Assume from now on that $X$ is geometrically connected and that the set $X(\mathbb{R})$ of $\mathbb{R}$-rational points is Zariski dense in $X$. The Galois group $G=\operatorname{Gal}(\mathbb{C} / \mathbb{R})$ acts on $\operatorname{Pic}\left(X_{\mathbb{C}}\right)$, and the Hochschild-Serre sequence $H^{i}\left(G, H_{\text {èt }}^{j}\left(X_{\mathbb{C}}, \mathbb{G}_{m}\right)\right) \Rightarrow H_{\text {ét }}^{i+j}\left(X, \mathbb{G}_{m}\right)$ implies that the natural map $\operatorname{Pic}(X) \rightarrow \operatorname{Pic}\left(X_{\mathbb{C}}\right)^{G}$ is an isomorphism.

Let $X_{1}, \ldots, X_{m}$ be the irreducible components of $X$, and let $J$ be the generalized Jacobian of $X$. Then $J$ is a connected algebraic group over $\mathbb{R}$, and we have the exact sequence

$$
0 \rightarrow J(\mathbb{C}) \rightarrow \operatorname{Pic}\left(X_{\mathbb{C}}\right) \rightarrow \mathbb{Z}^{m} \rightarrow 0
$$

where the last map is given by the partial degrees [2]. The Galois action on this sequence gives the exact sequence

$$
0 \rightarrow J(\mathbb{R}) \rightarrow \operatorname{Pic}(X) \rightarrow \mathbb{Z}^{m} \rightarrow 0
$$

(the last map is surjective since every component $X_{i}$ contains a nonsingular $\mathbb{R}$-point of $X$ ) and the isomorphism

$$
H^{1}(\mathbb{R}, J) \stackrel{\sim}{\rightarrow} H^{1}\left(G, \operatorname{Pic}\left(X_{\mathbb{C}}\right)\right)
$$

(writing $H^{1}(\mathbb{R}, J):=H^{1}(G, J(\mathbb{C}))$ as usual). 
Given an abelian group $M$, we denote by ${ }_{n} M=\operatorname{ker}(M \stackrel{n}{\longrightarrow} M)$ the $n$-torsion subgroup of $M$, for $n \in \mathbb{N}$. For any $G$-module $M$ there is a natural map ${ }_{2} M^{G} \rightarrow$ $H^{1}(G, M)$. We consider this map for $M=\operatorname{Pic}\left(X_{\mathbb{C}}\right)$ :

Proposition 2.3. The natural $\operatorname{map}_{2} \operatorname{Pic}(X) \rightarrow H^{1}\left(G, \operatorname{Pic}\left(X_{\mathbb{C}}\right)\right)$ is surjective.

Proof. The $\operatorname{map}_{2} J(\mathbb{R}) \rightarrow H^{1}(\mathbb{R}, J)$ is surjective, see [12], proof of Lemma 2.3(b). So the assertion follows from the commutative diagram



in which the right hand vertical arrow is surjective, see above.

2.4. Let $C(X)$ denote the set of connected components of $X(\mathbb{R})$, and let the finite abelian group $A_{X}$ be defined by the exact sequence

$$
1 \rightarrow\{ \pm 1\} \rightarrow\{ \pm 1\}^{C(X)} \rightarrow A_{X} \rightarrow 1
$$

where the first map is the diagonal embedding. We define a homomorphism

$$
\psi: H^{1}\left(G, \operatorname{Pic}\left(X_{\mathbb{C}}\right)\right) \rightarrow A_{X}
$$

as follows (cf. [12] 2.2). Given a Weil divisor $D$ on $X_{\mathbb{C}}$ with nonsingular support and with $D+\bar{D} \sim 0$, there is a rational function $g \in \mathbb{C}(X)^{*}$ with $\operatorname{div}(g)=D+\bar{D}$, and $g$ can be chosen to lie in $\mathbb{R}(X)^{*}$. Therefore $g$ has even order in every point of $X_{\text {reg }}(\mathbb{R})$ and is invertible around $X_{\text {sing }}(\mathbb{R})$. So there exists a sign tuple $\varepsilon \in\{ \pm 1\}^{C(X)}$ such that $\varepsilon(\xi) g(\xi) \geq 0$ for every $\xi \in X(\mathbb{R})$ where $g$ is defined. Since $g$ depends on $D$ only up to a factor in $\mathcal{O}(X)^{*}=\mathbb{R}^{*}$, this gives a well-defined element of $A_{X}$, which does not change if $D$ gets replaced by an equivalent Weil divisor on $\left(X_{\mathbb{C}}\right)_{\text {reg. }}$. So the map $\psi$ that sends the class of $D$ to $\pm \varepsilon \in A_{X}$ is a well-defined homomorphism.

Proposition 2.5. The map $\psi: H^{1}\left(G, \operatorname{Pic}\left(X_{\mathbb{C}}\right)\right) \rightarrow A_{X}$ is surjective.

Proof. Let a sign distribution $\varepsilon \in\{ \pm 1\}^{C(X)}$ be given. By Weierstraß approximation there exists a rational function $f \in \mathbb{R}(X)^{*}$ without real zeros or poles that is invertible around every singular point of $X$, and such that $\varepsilon(\xi) f(\xi) \geq 0$ holds for every $\xi \in X(\mathbb{R})$. The $\operatorname{divisor} \operatorname{div}(f)$ of $f$ on $X_{\mathbb{C}}$ is a $G$-invariant Weil divisor on $\left(X_{\mathbb{C}}\right)_{\text {reg }}$ without real points. Hence it can be written $\operatorname{div}(f)=D+\bar{D}$ with $D \in \operatorname{Div}\left(X_{\mathbb{C}}\right)$. The class defined by $D$ in $H^{1}\left(G, \operatorname{Pic}\left(X_{\mathbb{C}}\right)\right)$ maps to $\pm \varepsilon$ under $\psi$.

When $X$ is nonsingular, the map $\psi$ in 2.5 is even bijective, see [12] Remark 2.2.

Corollary 2.6. The map $\sigma:{ }_{2} \operatorname{Pic}(X) \rightarrow A_{X}$ which is the composite of the map 2.3 and of $\psi$ (see 2.4) is surjective.

Definition 2.7. A 2-torsion class $\tau \in{ }_{2} \operatorname{Pic}(X)$ will be called definite if it lies in the kernel of the natural map $\sigma:{ }_{2} \operatorname{Pic}(X) \rightarrow A_{X}$ (see 2.6).

So if $D$ is a Weil divisor on $X$ with nonsingular support and with $2 D \sim 0$, the class $[D]$ is definite if and only if there exists a rational function $g \in \mathbb{R}(X)^{*}$, invertible around $X_{\text {sing }}$, with $\operatorname{div}(g)=2 D$ and with $g \geq 0$ on $X(\mathbb{R})$ (where $g$ is defined).

For plane cubics we determine the definite 2-torsion classes explicitly: 
Proposition 2.8. Let $X$ be a reduced plane cubic curve with $X(\mathbb{R})$ Zariski dense in $X$. If every singularity of $X$ is a node, there exists a unique nonzero class in ${ }_{2} \operatorname{Pic}(X)$ that is definite. Otherwise the only definite class in ${ }_{2} \operatorname{Pic}(X)$ is zero.

Proof. When $X$ is nonsingular, the assertion says $|\operatorname{ker}(\sigma)|=2$ and is a particular case of [12, Lemma 2.3(c). (The map $\sigma$ considered here is identified with the map $\bar{\phi}$ studied in loc. cit., via the natural isomorphism $H^{1}(\mathbb{R}, J) \stackrel{\sim}{\rightarrow} A_{X}$ observed in [12] Remark 2.2.)

For singular $X$ the claim is proved by computing the group $J(\mathbb{R})=\operatorname{Pic}_{0}(X)$ and using 2.6. In more detail (compare 2] 9.2 for computation of the Picard groups):

When $X$ is irreducible, $\operatorname{Pic}_{0}(X)=J(\mathbb{R})$ is isomorphic to $\mathbb{R}, \mathbb{R}^{*}$ or $\mathbb{C}^{*} / \mathbb{R}^{*}$, depending on whether $X$ has a cusp, a node or an acnode, respectively. Note that $\left|A_{X}\right|=1$ in the first two cases and $\left|A_{X}\right|=2$ in the third. When $X$ is the union of a conic $Q$ and a line $L$, we have $\operatorname{Pic}_{0}(X) \cong \mathbb{R}^{*}, \mathbb{R}$ or $\mathbb{C}^{*} / \mathbb{R}^{*}$, depending on whether $|Q(\mathbb{R}) \cap L(\mathbb{R})|=2,1$ or 0 . Again we have $\left|A_{X}\right|=1$ in the first two cases and $\left|A_{X}\right|=2$ in the last. When $X$ is the union of three lines, $\operatorname{Pic}_{0}(X) \cong \mathbb{R}$ or $\mathbb{R}^{*}$, according to whether the lines meet in a common point or not, and $\left|A_{X}\right|=1$ in either case. Since $\sigma$ is surjective by Corollary 2.6. this implies the assertion in each case.

Notation 2.9. Let $X$ be a reduced plane cubic with $X(\mathbb{R})$ Zariski dense in $X$ whose only singularities are nodes, and let $\tau$ be the unique definite nonzero 2 -torsion class in $\operatorname{Pic}(X)$ (see Proposition 2.8). We write

$$
\tau_{X}:=\tau+\left[\mathcal{O}_{X}(3)\right] \in \operatorname{Pic}(X) .
$$

\section{NiNE REAL ZEROS}

Let $S \subseteq \mathbb{P}^{2}(\mathbb{R})$ be any set of nine points. There is at least one cubic $X$ passing through $S$, i.e., $\operatorname{dim} I_{3}(S) \geq 1$. If there exists a psd and non-sos sextic through $S$, we show in this section that $X$ is unique, and that $S$ gives rise to the divisor class $\tau_{X}$ on $X$ (see 2.9). In the next section we will prove the converse.

3.1. For $T \subseteq \mathbb{P}^{2}(\mathbb{R})$ a finite set, recall that $I_{d}(m T) \subseteq \mathbb{R}\left[x_{0}, x_{1}, x_{2}\right]$ is the space of forms of degree $d$ that have multiplicity $\geq m$ at every point of $T$. The psd forms of degree $2 d$ that vanish in $T$ form a face of the cone $P_{2 d}$ denoted

$$
P_{2 d}(T)=\left\{p \in P_{2 d}:\left.p\right|_{T}=0\right\},
$$

and we put $\Sigma_{2 d}(T)=\Sigma_{2 d} \cap P_{2 d}(T)$. The forms in $\Sigma_{2 d}(T)$ are the sums of squares of forms in $I_{d}(T)$.

The following facts are certainly well known, and are recorded for reference:

Proposition 3.2. Let $T \subseteq \mathbb{P}^{2}(\mathbb{R})$ be any set of 8 points, no 4 on a line and no 7 on a conic.

(a) $\operatorname{dim} I_{3}(T)=2$, and almost every cubic in $I_{3}(T)$ is nonsingular.

(b) $\operatorname{dim} I_{6}(2 T)=4$ and $\operatorname{dim} I_{3}(T)^{2}=3$.

(c) Every psd sextic in $I_{3}(T)^{2}$ is a sum of two squares of cubics. In particular, $\Sigma_{6}(T)=I_{3}(T)^{2} \cap P_{6}(T)$.

Here and in the sequel, $I_{3}(T)^{2}$ denotes the space of sextic forms spanned by the products $f_{1} f_{2}$ with $f_{1}, f_{2} \in I_{3}(T)$. 
Proof. (a) is classical, see e.g. [5] V.4.4. (b) Let $I_{3}(T)=\operatorname{span}\left(f, f^{\prime}\right)$ with $f$ nonsingular. Then $I_{3}(T)^{2}=\operatorname{span}\left(f^{2}, f f^{\prime}, f^{\prime 2}\right)$ has dimension 3 since $f, f^{\prime}$ are algebraically independent. Being singular in the points of $T$ imposes $8 \cdot 3=24$ linear conditions on a sextic, showing $\operatorname{dim} I_{6}(2 T) \geq 4$. For the reverse inequality consider the divisor $D=\sum_{P \in T} P$ on $X=V(f)$, and let $M \in X(\mathbb{R})$ be the point with $\operatorname{div}_{X}\left(f^{\prime}\right)=D+M$. If $g \in I_{6}(2 T)$ is prime to $f$ and satisfies $\operatorname{div}_{X}(g) \geq 2 D+M$ then $\operatorname{div}_{X}(g)=2(D+M)$, implying that $g \in I_{3}(T)^{2}$. This shows that $I_{3}(T)^{2}$ has codimension $\leq 1$ in $I_{6}(2 T)$. (c) Let $g \in I_{3}(T)^{2}$ be a psd sextic form. Then $g=q\left(f, f^{\prime}\right)$ is a quadratic form in $f$ and $f^{\prime}$, and the binary quadratic form $q\left(t_{0}, t_{1}\right)$ is necessarily positive definite, as can be seen locally around any transversal intersection point of $f$ and $f^{\prime}$. Hence $q$ is a sum of two squares from $I_{3}(S)$.

3.3. Let $g \in \mathbb{R}\left[x_{0}, x_{1}, x_{2}\right]=\mathbb{R}[\mathrm{x}]$ be a form of degree 6 that is psd. If $g$ is reducible over $\mathbb{R}$ then $g$ is sos, since every ternary psd form of degree $\leq 4$ is sos (Hilbert [6]). Moreover $\left|V_{\mathbb{R}}(g)\right| \leq 5$ or $\left|V_{\mathbb{R}}(g)\right|=\infty$ in this case. Now assume that $g$ is irreducible over $\mathbb{R}$. Since every real zero of $g$ is a singular point of the curve $V(g)$, there can be at most 10 real zeros (and at most 9 when $g$ is reducible over $\mathbb{C}$ ). We assume that $g$ has at least 9 real zeros, and we fix a set $S \subseteq V_{\mathbb{R}}(g)$ with $|S|=9$.

Lemma 3.4. Let $g \in \mathbb{R}[\mathrm{x}]$ be a psd sextic form with $\left|V_{\mathbb{R}}(g)\right|<\infty$, and let $S \subseteq V_{\mathbb{R}}(g)$ with $|S|=9$. Any cubic through $S$ is reduced with Zariski dense $\mathbb{R}$-points, and is nonsingular in the points of $S$. Moreover,

(a) if $\operatorname{dim} I_{3}(S)=2$ then $g$ is a sum of two squares of cubic forms, hence reducible over $\mathbb{C}$,

(b) otherwise $\operatorname{dim} I_{3}(S)=1$, and $g$ is absolutely irreducible and not a sum of squares in $\mathbb{R}[\mathrm{x}]$.

Proof. $g$ is irreducible, so $S$ contains no 4 points on a line and no 7 points on a conic. By 3.2 (a) we have $\operatorname{dim} I_{3}(S) \in\{1,2\}$.

Let $X=V(f)$ be a cubic through $S$. It is clear that $X$ is reduced and $X(\mathbb{R})$ is Zariski dense in $X$. Moreover $S$ cannot contain a singular point of $X$, since otherwise the intersection product of $X$ and $Y=V(g)$ would satisfy $X . Y>2 \cdot 9=$ 18 , contradicting that $Y$ is irreduzible.

If $\operatorname{dim} I_{3}(S)=2$ and $I_{3}(S)$ is spanned by $f$ and $f^{\prime}$, then $f, f^{\prime}$ are relatively prime and $S$ is the set of their common zeros. It follows that $g$ is a quadratic form in $f, f^{\prime}$, and by 3.2 (c), $g$ is a sum of two squares from $I_{3}(S)$.

On the other hand, when $X=V(f)$ is the unique cubic through $S$, the only sos sextics vanishing in $S$ are multiples of $f^{2}$. So $g$ is not a sum of squares in this case, and is therefore irreducible over $\mathbb{C}$.

3.5. Assume now that the psd sextic $g$ is not a sum of squares and has at least 9 real zeros. Fix a subset $S \subseteq V_{\mathbb{R}}(g)$ with $|S|=9$. By 3.4 there is a unique cubic $X=V(f)$ through $S$, and $X$ is reduced with $X(\mathbb{R})$ Zariski dense in $X$ and $S \subseteq X_{\text {reg }}(\mathbb{R})$. However, $X$ may be singular or even reducible. The Weil divisor $D=\sum_{P \in S} P$ on $X_{\text {reg }}$ defines a class in $\operatorname{Pic}(X)$. Let $L \in \operatorname{Div}(X)$ be the divisor of a line section of $X$.

Proposition 3.6. The divisor class $[D-3 L]$ in $\operatorname{Pic}(X)$ is a nonzero 2-torsion class and is definite 2.4.

$$
\text { So }[D]=\tau_{X} \text {, see Notation } 2.9 \text {, }
$$


Proof. From $\operatorname{div}_{X}(g) \sim 6 L$ and $\operatorname{div}_{X}(g)=2 D$ it follows that $2(D-3 L) \sim 0$ on $X$. Assuming $D \sim 3 L$ there would exist a cubic form $f^{\prime}$, prime to $f$, with $\operatorname{div}_{X}\left(f^{\prime}\right)=D$, contradicting $I_{3}(S)=\mathbb{R} f$ (3.4). Since $2(D-3 L)$ is the divisor of the psd rational function $g / l^{6}$ on $X$, it is clear that $\tau$ is definite, see Definition 2.7.

For a convenient way of speaking we introduce the following terminology:

Definition 3.7. A set $S \subseteq \mathbb{P}^{2}(\mathbb{R})$ will be said to be admissible if $|S|=9$, and if there exists a reduced plane cubic $X$ with $S \subseteq X_{\text {reg }}(\mathbb{R})$ for which $\sum_{P \in S}[P]=\tau_{X}$ in $\operatorname{Pic}(X)$.

We have therefore proved:

Corollary 3.8. Let $g$ be a psd sextic that is not a sum of squares. Then any set $S \subseteq V_{\mathbb{R}}(g)$ with $|S|=9$ is admissible.

Next we discuss properties of admissible sets.

Proposition 3.9. Let $S \subseteq \mathbb{P}^{2}(\mathbb{R})$ be an admissible set, let $f \neq 0$ be a cubic form vanishing on $S$, and let $X=V(f)$.

(a) $I_{3}(S)=\mathbb{R} f$, so $X$ is uniquely determined by $S$.

(b) $X$ is reduced, $S \subseteq X_{\mathrm{reg}}(\mathbb{R})$, and $X(\mathbb{R})$ is Zariski dense in $X$.

(c) For every irreducible component $X^{\prime}$ of $X$ we have $\left|S \cap X^{\prime}(\mathbb{R})\right|=3 d^{\prime}$ where $d^{\prime}=\operatorname{deg}\left(X^{\prime}\right)$.

In view of (a), we call $X=V(f)$ the cubic associated to $S$.

Proof. Since $S$ is admissible, there exists a reduced cubic as in Definition 3.7. In view of (a), it suffices to prove (a)-(c) for this cubic. So we may assume that $X=$ $V(f)$ is reduced with $S \subseteq X_{\mathrm{reg}}(\mathbb{R})$, and that $\left[\mathcal{O}_{X}(3)\right]-\sum_{P \in S}[P]$ is a 2-torsion class in $\operatorname{Pic}(X)$. Let $X^{\prime} \subseteq X$ be an irreducible component, and let $D^{\prime}=\sum_{P \in S \cap X^{\prime}(\mathbb{R})} P \in$ $\operatorname{Div}\left(X^{\prime}\right)$. Then $2\left[D^{\prime}\right]=\left[\mathcal{O}_{X^{\prime}}(6)\right]$ in $\operatorname{Pic}\left(X^{\prime}\right)$, which implies (c). In particular, every irreducible component of $X$ contains a nonsingular $\mathbb{R}$-point. Hence $X(\mathbb{R})$ is Zariski dense in $X$. It remains to prove (a). Let $h \in I_{3}(S)$, and assume $h \notin \mathbb{R} f$. Writing $g=\operatorname{gcd}(f, h)$ and $f=f^{\prime} g, h=h^{\prime} g$, the degree $d^{\prime}:=\operatorname{deg}\left(f^{\prime}\right)=\operatorname{deg}\left(h^{\prime}\right)$ satisfies $d^{\prime} \geq 1$. Put $X^{\prime}=V\left(f^{\prime}\right)$ and $S^{\prime}=S \cap X^{\prime}(\mathbb{R})$. Each point of $S$ lies on a single irreducible component of $X$, therefore $h^{\prime}$ vanishes on $S^{\prime}$. Therefore by (c), $h^{\prime}$ has at least $3 d^{\prime}$ different zeros on $X^{\prime}$. On the other hand the number of zeros of $h^{\prime}$ on $X^{\prime}$ is $d^{\prime 2}$ by Bézout's theorem, since $\operatorname{gcd}\left(f^{\prime}, h^{\prime}\right)=1$. It follows that $d^{\prime}=3$, and so $\operatorname{gcd}(f, h)=1$. Hence $D=\operatorname{div}_{X}(h)$, which implies $[D]=\left[\mathcal{O}_{X}(3)\right]$, contradicting $[D]=\tau_{X}$. So the assumption was false, proving (a).

Remark 3.10. Let $X$ be the cubic associated to the admissible set $S$. According to Proposition 2.8, there are four possibilities for $X$ : Either $X$ is nonsingular, or else irreducible with a node, or else the union of a line and a conic intersecting transversally in two $\mathbb{R}$-points, or else a triangle (union of three lines without a common point).

Remarks 3.11. The admissibility condition can be characterized in more elementary geometric terms, as follows. Let $X=V(f)$ be a plane cubic curve, and assume for simplicity that $X$ is irreducible.

1. Let $S \subseteq X_{\text {reg }}(\mathbb{R})$ be a set with $|S|=9$. Choose a point $P \in S$ (it does not matter which one), and let $X^{\prime}=V\left(f^{\prime}\right)$ be a cubic through $S^{\prime}=S \backslash\{P\}$, different from $X$. Let $M$ be the 9 th point of intersection of $X$ and $X^{\prime}$, a nonsingular point 
of $X$, and let $t_{M}$ resp. $t_{P}$ be the tangent of $X$ at $M$ resp. $P$. Then $S$ is admissible if and only if $M \neq P$, the tangents $t_{M}$ and $t_{P}$ meet in a point of $X$, and the product $t_{M} t_{P}$ is semidefinite on $X(\mathbb{R})$.

Indeed, by 3.9)(a), $S$ is admissible if and only if $X$ and $S$ satisfy the property in 3.7. Consider the divisors $D=\sum_{Q \in S} Q$ and $D^{\prime}=M+\sum_{Q \in S^{\prime}} Q$ on $X$, and let $L=\operatorname{div}(l) \in \operatorname{Div}(X)$ be the divisor of a linear form $l$ with $l \nmid f$. Then $D^{\prime} \sim 3 L$, so $D-3 L \nsim 0$ means $M \neq P$. On the other hand, $2(D-3 L) \sim 0$ means $2 M \sim 2 P$ on $X$, which says that $t_{M}$ and $t_{P}$ meet on $X$. Assuming this condition, $\frac{t_{P}}{t_{M}}$ is a rational function on $X$ whose divisor is $2(P-M)$. So the rational function

$$
\frac{f^{\prime 2}}{l^{6}} \cdot \frac{t_{P}}{t_{M}}
$$

on $X$ has divisor $2(D-3 L)$, proving the characterization of the definiteness condition claimed above.

2. Let $X$ be an irreducible plane cubic and $T \subseteq X_{\text {reg }}(\mathbb{R})$ a set with $|T|=8$. There can be at most one point $Q \in X(\mathbb{R})$ for which the set $T \cup\{Q\}$ is admissible. In fact, such $Q$ exists if and only if $X$ is nonsingular or has a node, and if the unique point $Q \in X_{\text {reg }}(\mathbb{R})$ with $\left[Q+\sum_{P \in T} P\right]=\tau_{X}$ satisfies $Q \notin T$. In this case, $T \cup\{Q\}$ is admissible.

The previous remark shows how to construct $Q$ geometrically. Indeed, let $M$ be as before, and let $N$ be the third intersection point of $t_{M}$ with $X$. When $X$ is nonsingular or has a node, there is a unique tangent $t \neq t_{M}$ to $X$ that passes through $N$ for which $t_{M} t$ is semidefinite on $X(\mathbb{R})$. This tangent $t$ touches $X$ in $Q$.

\section{Constructing nOnnegative SeXtics With Nine Real Zeros}

In this section we prove the converse of Corollary 3.8. As before we work in the projective plane $\mathbb{P}^{2}$ over $\mathbb{R}$ and write $\mathrm{x}=\left(x_{0}, x_{1}, x_{2}\right)$.

We start with two technical lemmas.

Lemma 4.1. Let $X=V(f)$ be a reduced plane cubic, and let $T \subseteq X_{\mathrm{reg}}(\mathbb{R})$ be a set with $|T|=8$ or 9 . If $|T|=9$, assume that $I_{3}(T)=\mathbb{R} f$. Let $g$ be a sextic form with $\operatorname{gcd}(f, g)=1$ such that $i_{P}(f, g) \geq 2$ for every $P \in T$. Then there exists a cubic form $p$ such that the sextic $g+p f$ is singular in every point of $T$.

Proof. The sextic $g$ shows that no 4 points of $T$ are on a line and no 7 on a conic. Therefore $\operatorname{dim} I_{3}(T)=2$ in the case $|T|=8$ (3.2). Let $f_{x_{i}}:=\partial f / \partial x_{i}(i=0,1,2)$ denote the partial derivatives. From the assumptions it follows that

$$
\operatorname{rk}\left(\begin{array}{lll}
f_{x_{0}} & f_{x_{1}} & f_{x_{2}} \\
g_{x_{0}} & g_{x_{1}} & g_{x_{2}}
\end{array}\right)(P)=1
$$

at every point $P \in T$. Hence there exists a unique section $\lambda \in \Gamma(T, \mathcal{O}(3))$ such that $g_{x_{i}}-\lambda f_{x_{i}}$ vanishes on $T(i=0,1,2)$. The natural map $\Gamma\left(\mathbb{P}^{2}, \mathcal{O}(3)\right) \rightarrow \Gamma(T, \mathcal{O}(3))$ is surjective since its kernel $I_{3}(T)$ has dimension $10-|T|$. Hence there exists a cubic form $p$ which restricts to $\lambda$ on $S$. The sextic $q-p f$ is singular in the points of $T$.

Lemma 4.2. Let $X=V(f)$ be a plane curve of degree $d$, let $g$ be a form of degree $2 d$, and let $P \in X_{\mathrm{reg}}(\mathbb{R})$. Suppose that $g$ is singular at $P$ and $i_{P}(f, g)=2$. If $g \geq 0$ locally around $P$ on $X(\mathbb{R})$, there exists $t>0$ such that $g+t f^{2} \geq 0$ locally around $P$ in the plane. 
Proof. Given the other assumptions, the condition $i_{P}(f, g)=2$ means that the tangent of $X=V(f)$ at $P$ is not a tangent of the curve $V(g)$ at $P$. Therefore, for sufficiently large $t>0, g+t f^{2}$ has positive definite Hessian at $P$ in local affine coordinates, proving the lemma.

In the following let always $S \subseteq \mathbb{P}^{2}(\mathbb{R})$ be an admissible set (Definition 3.7), and let $X=V(f)$ be the cubic associated to $S$, see 3.9. Writing $D=\sum_{P \in S} P \in \operatorname{Div}(X)$ we have $[D]=\tau_{X}$.

Lemma 4.3. There exists a sextic form $q$ with $\operatorname{gcd}(f, q)=1$ that is singular in the points of $S$.

Proof. Since $2[D]=\left[\mathcal{O}_{X}(6)\right]$, there exists a sextic form $g$ with $\operatorname{gcd}(f, q)=1$ such that $\operatorname{div}_{X}(q)=2 D$. We can apply Lemma 4.1 since $I_{3}(S)=\mathbb{R} f$ holds by 3.9. (a). This proves the lemma.

Lemma 4.4. Let $q$ be a sextic as in Lemma 4.3. There exists a real number $t>0$ and a choice of sign \pm such that $t f^{2} \pm q$ is psd $\left(\right.$ on $\left.\mathbb{P}^{2}(\mathbb{R})\right)$.

Proof. The sextic $q$ has $\operatorname{div}_{X}(q)=2 D$. Let $l$ be a linear form not dividing $f$, and let $L=\operatorname{div}(l) \in \operatorname{Div}(X)$. Since the class $[D-3 L]=\tau_{X} \in{ }_{2} \operatorname{Pic}(X)$ is definite by assumption, the rational function $q / l^{6}$ on $X$ is semidefinite on $X(\mathbb{R})$. After replacing $q$ with $-q$ if necessary, we can assume that $q \geq 0$ on $X(\mathbb{R})$. For any point $P \in S$ the local intersection number $i_{P}(f, q)=2$, so by Lemma 4.2 there exists $t>0$ such that $t f^{2}+q$ is psd around $P$. Now the assertion follows from the following Lemma 4.5, which is an easy compactness argument.

Lemma 4.5. Let $p, q \in \mathbb{R}[\mathrm{x}]$ be forms of the same even degree. Assume that $p$ is $p s d$, and that for every real zero $P$ of $p$ there is a real number $t$ such that $q+t p$ is nonnegative in a neighborhood of $P$. Then there exists $t \in \mathbb{R}$ such that the form $q+t p$ is $p s d$.

Proof. Since $\mathbb{P}^{2}(\mathbb{R})$ is compact, there exist finitely many open sets $U_{i} \subseteq \mathbb{P}^{2}(\mathbb{R})$ and real numbers $t_{i}(i=1, \ldots, r)$, such that $\mathbb{P}^{2}(\mathbb{R})$ is covered by the $U_{i}$ and $q+t_{i} p \geq 0$ on $U_{i}$ for every $i$. It suffices to take $t=\max \left\{t_{1}, \ldots, t_{r}\right\}$.

The next result completes and summarizes the discussion:

Theorem 4.6. Let $S \subseteq \mathbb{P}^{2}(\mathbb{R})$ be an admissible set with associated cubic $X=V(f)$ (see 3.9). The space $I_{6}(2 S)$ of sextics singular in $S$ has dimension 2 . The psd sextics vanishing in $S$ form a 2-dimensional cone $P_{6}(S)$ in $I_{6}(2 S)$, while $\Sigma_{6}(S)=\mathbb{R}_{+} f^{2}$ has dimension 1. There exists a unique (up to positive scaling) sextic in $P_{6}(S) \backslash \Sigma_{6}(S)$ that is extreme in the psd cone.

Proof. By Lemma 4.4 there exists a psd sextic $q$ with $(f, q)=1$ that vanishes in $S$. Such $q$ cannot be a sum of squares since $q=\sum_{i} q_{i}^{2}$ implies $q_{i} \in I_{3}(S)$, and since $I_{3}(S)=\mathbb{R} f$ by $\left[3.9\right.$ (a). So it only remains to show that $I_{6}(2 S)$ is spanned by $q$ and $f^{2}$. The argument is slightly technical since $X$ may be reducible. Let $0 \neq h \in I_{6}(2 S)$, let $f_{1}=\operatorname{gcd}(f, h)$, and write $f=f_{1} f_{2}$ and $h=f_{1} h_{2}$. Let $d_{i}=\operatorname{deg}\left(f_{i}\right)(i=1,2)$, then we have $d_{1}+d_{2}=3$ and $\operatorname{deg}\left(h_{2}\right)=6-d_{1}=3+d_{2}$. Let $X_{2}:=V\left(f_{2}\right)$ and $S_{2}:=S \cap X_{2}(\mathbb{R})$. Then $\left|S_{2}\right|=3 d_{2}$ by $3.9(\mathrm{c})$. Since $\operatorname{gcd}\left(f_{2}, h_{2}\right)=1$, the form $h_{2}$ has precisely $\left(6-d_{1}\right) d_{2}$ zeros on $X_{2}$ by Bézout. On the other hand, $h_{2}$ has multiplicity $\geq 2$ at each point of $S_{2}$, and so $h_{2}$ has at least $2\left|S_{2}\right|=6 d_{2}$ zeros on $X_{2}$. It follows that $d_{1}=0$ or $d_{2}=0$. If $d_{1}=0$ then $\operatorname{gcd}(f, h)=1$, and we 
conclude $\operatorname{div}_{X}(h)=2 D=\operatorname{div}_{X}(q)$, so $h=c q+f f^{\prime}(\bmod f)$ with some $c \in \mathbb{R}^{*}$ and some cubic form $f^{\prime}$. Necessarily $f^{\prime} \in I_{3}(S)$, so $h \in \mathbb{R} q+\mathbb{R} f^{2}$. On the other hand, if $d_{2}=0$ then $h=f f^{\prime}$ with some cubic form $f^{\prime}$, and using again the argument just given we conclude $h \in \mathbb{R} f^{2}$.

Corollary 4.7. A set $S \subseteq \mathbb{P}^{2}(\mathbb{R})$ with $|S|=9$ is admissible if and only if there exists a psd sextic vanishing in $S$ that is not a sum of squares.

Proof. Follows from 3.8 and 4.6 .

Notation 4.8. Let $S \subseteq \mathbb{P}^{2}(\mathbb{R})$ be an admissible set. We denote the unique (up to scaling) extreme psd form in $P_{6}(S)$ by $q_{S}$, bearing in mind the ambiguity by a scalar factor.

Remark 4.9. In the situation of 4.6, fix any $q \in I_{6}(2 S)$ with $q \notin \mathbb{R} f^{2}$. Replacing $q$ with $-q$ if necessary we can assume $q \geq 0$ on $X(\mathbb{R})$. Then there exists a minimal real number $s$ for which the form $q_{s}:=q+s f^{2}$ is psd, by Lemma 4.4 and since the psd cone is closed. We have $q_{s}=q_{S}$, and the cone $P_{6}(S)$ is generated by $f^{2}$ and $q_{S}$. The number $s$ resp. the extreme form $q_{S}$ can be found numerically by solving a semidefinite program, since $q_{t}$ is psd if and only if there exists a quadratic form $p \neq 0$ for which $p q_{t}$ is a sum of squares (Hilbert [7). Other ways of finding $q_{S}$ are discussed below (Remark 4.11. Corollary 4.19 and Remark 4.20).

Proposition 4.10. Let $S$ be admissible with associated cubic $X=V(f)$, let $I_{6}(2 S)=\operatorname{span}\left(f^{2}, q\right)$ with $q \geq 0$ on $X(\mathbb{R})$. Write $q_{t}:=q+t f^{2}$ for $t \in \mathbb{R}$, and let $s \in \mathbb{R}$ be the minimal number for which $q_{s}$ is psd $\left(\right.$ so $\left.q_{s}=q_{S}\right)$.

(a) For each point $P \in S$ there exists a unique number $t(P) \in \mathbb{R}$ for which $P$ is neither a node nor an acnode of the sextic $q_{t(P)}=0$.

(b) $s \geq \max \{t(P): P \in S\}$.

(c) For generic choice of $S$ on $X$ the inequality (b) is strict, and the extreme psd sextic $q_{S}$ has a tenth real zero $P$ with $P \notin X(\mathbb{R})$.

(d) The sextic $q_{S}=0$ is a (geometrically) rational curve.

Proof. (a) Let $x, y$ be local affine coordinates centered at $P$ such that $X=V(f)$ has tangent $x=0$ at $P$. Fix a linear form $l$ with $l(P) \neq 0$ such that $f / l^{3}=x+$ (higher order terms) at $P$. Then $q / l^{6}=a x^{2}+b x y+c y^{2}+$ (higher order terms) at $P$ with suitable $a, b, c \in \mathbb{R}$, and $c>0$ since $f$ and $q$ have no common tangent at $P$ and $q \geq 0$ on $X(\mathbb{R})$. Therefore $t(P)$ is determined by the condition $b^{2}-4 c(a+t(P))=0$. The singularity $P$ of $q_{t}$ is a node for $t<t(P)$ and an acnode for $t>t(P)$. Therefore $q_{t}$ is indefinite for $t<t(P)$, proving (a) and (b). Generically, $P$ will be a cusp of the sextic $q_{t(P)}$, and thus $q_{t(P)}$ will be indefinite, showing $s>\max \{t(P): P \in S\}$. Therefore $q_{s}=q_{S}$ must have an additional tenth real zero in this case, proving (c). In the non-generic case $\left|V_{\mathbb{R}}\left(q_{S}\right)\right|=9$, one of the points $P \in S$ is an $A_{3}$-singularity (with complex conjugate branches) of $q_{S}$. In either case it is clear that $q_{S}$ is a rational curve.

Note that assertion (d) was already known from results in [1, see Remark 4.16]2 below.

Remark 4.11. Let $S$ be an admissible set. We describe an effective algorithm to (precisely) compute the extreme sextic $q_{S}$, and possibly its 10th zero. Consider the assumptions of Proposition 4.10. For almost all values of $t \in \mathbb{C}$, the sextic $q_{t}$ has 
a complex node at each point of $S$, and no other singularities. Let $\mathcal{E}$ be the finite set of numbers $t \in \mathbb{C}$ for which $q_{t}$ has a higher singularity at some point of $S$, or a singularity outside $S$. The set $\mathcal{E}$ can be found explicitly via elimination theory, e.g. using a computer algebra system. Clearly, $\mathcal{E}$ contains the numbers $s$ and $t(P)$ from 4.10. In fact, it is easy to see that $s$ is precisely the largest real number in $\mathcal{E}$. Indeed, this follows from 4.10(b), since for real $t>s$ the sextic $q_{t}$ is irreducible and cannot have an additional singularity $P \notin S$ (necessarily $P$ would have to be real).

Remark 4.12. "Hilbert's method" for constructing psd non-sos sextics, as formalized in 9 , starts with a set $T \subseteq \mathbb{P}^{2}(\mathbb{R})$ of 8 points such that the pencil $I_{3}(T)$ has a 9 th base point $E \notin T$. Choose a basis $f_{1}, f_{2}$ of $I_{3}(T)$ and a sextic $g \in I_{6}(2 T)$ with $g \notin I_{3}(T)^{2}$, i.e. $g(E) \neq 0$ (c.f. 3.2). Then for large $t>0$, the sextic $q=g+t\left(f_{1}^{2}+f_{2}^{2}\right)$ is psd and not sos. Generically, $q$ has a 9 th real zero $P$, but there is no control of $P$, and $q$ is usually not extreme, not even when $t$ is chosen minimally.

We now consider collections of 8 points in the plane.

Theorem 4.13. Let $T \subseteq \mathbb{P}^{2}(\mathbb{R})$ be any set with $|T|=8$ and no 4 points on a line, no 7 on a conic. Then there exists a psd non-sos sextic that vanishes in $T$.

This extends results by Reznick. He used Hilbert's construction to prove the assertion when $T$ is "copacetic" (meaning that the 9th base point of $I_{3}(T)$ lies outside $T$ ), and derived from this the unconditional assertion for $|T|=7$. See $[9]$, Corollaries 4.2 and 4.4. Our proof doesn't use these results.

Proof. Let $I_{3}(T)$ be spanned by the nonsingular cubics $f$ and $f^{\prime}$, and let $E$ be their 9 th intersection point (which may lie in $T$ ). All divisors will be formed on the curve $X=V(f)$. Let $Q \in X(\mathbb{R})$ be the unique point with $\left[Q+\sum_{P \in T} P\right]=\tau_{X}$. When $Q \notin T$ then $T \cup\{Q\}$ is admissible, see Remark 3.11,2. Hence, in this case, there exists a psd non-sos sextic vanishing even in $T \cup\{Q\}$.

So we assume $Q \in T$ and write $T^{\prime}=T \backslash\{Q\}$. Note that $Q \neq E$. Let $D=$ $\sum_{P \in T^{\prime}} P \in \operatorname{Div}(X)$. We have $\operatorname{div}\left(f^{\prime}\right)=D+Q+E$ and $[D+2 Q]=\tau_{X}$. There exists a sextic form $g$, prime to $f$, with $\operatorname{div}(g)=2(D+2 Q)=2 D+4 Q$, and we have $g \geq 0$ on $X(\mathbb{R})$ by the definiteness condition on $\tau_{X}$. By Lemma 4.1 there exists a cubic form $h$ such that $g+f h$ is singular in every point of $T$. Replacing $g$ with $g+f h$ we can assume $g \in I_{6}(2 T)$. The $\operatorname{divisor} \operatorname{div}(g)$ shows that $g \notin I_{3}(T)^{2}$. In particular, adding to $g$ a form in $I_{3}(T)^{2}$ to $g$ cannot turn $g$ into a sum of squares.

Since $f$ and $g$ have local intersection index 2 at every point of $T^{\prime}=T \backslash\{Q\}$, there exists $c>0$ such that $g^{\prime}:=g+c f^{2}$ is locally psd around every point of $T^{\prime}$, by Lemma 4.2. For the form $g^{\prime \prime}:=g^{\prime}+f^{\prime 2}$, the intersection index with $f$ at the point $Q$ drops to 2 . Now we can apply Lemma 4.2 at the point $Q$, and then apply Lemma 4.5, to conclude that the sextic $g^{\prime \prime}+t f^{2}$ is psd for sufficiently large $t>0$.

Corollary 4.14. Let $T \subseteq \mathbb{P}^{2}(\mathbb{R})$ be any set with $|T|=8$, having no 4 points on a line, no 7 on a conic. The cone $P_{6}(T)$ in $I_{6}(2 T)$ has full dimension four, while $\Sigma_{6}(T)=P_{6}(T) \cap I_{3}(T)^{2}$ has dimension three.

Proof. Follows from Theorem 4.13 and Proposition 3.2 (c).

Remark 4.15. For most sets $T \subseteq \mathbb{P}^{2}(\mathbb{R})$ of 8 points (no 4 on a line, no 7 on a conic) we have seen that there exists a non-sos psd sextic $q$ that vanishes on $T$ plus an extra point outside $T$. It is clear that such $q$ exists if and only if $T$ has the following property: There exists a nonsingular cubic $X$ through $T$ for which the 
(unique) point $Q_{X} \in X(\mathbb{R})$ with $\left[Q_{X}+\sum_{P \in T} P\right]=\tau_{X}$ satisfies $Q_{X} \notin T$. We do not know if there exists a set $T$ for which this property fails.

\section{Remarks 4.16.}

1. The psd sextic $g$ is said to be exposed if any $g^{\prime} \in P_{6}$ with $V_{\mathbb{R}}(g) \subseteq V_{\mathbb{R}}\left(g^{\prime}\right)$ satisfies $g^{\prime} \in \mathbb{R}_{+} g$. Clearly such $g$ spans an extreme ray of $P_{6}$. The converse is not true in general, however Straszewicz's theorem (e.g. [1] Theorem 18.6) shows that every extreme form is the limit of a sequence of exposed forms. It is easy to see that a form $g \in P_{6} \backslash \Sigma_{6}$ is exposed if and only if $\left|V_{\mathbb{R}}(g)\right|=10$. Indeed, when $\left|V_{\mathbb{R}}(g)\right| \leq 9$ there is a cubic $p$ through $V_{\mathbb{R}}(g)$, so $p^{2}$ is another psd sextic vanishing on $V_{\mathbb{R}}(g)$. Conversely, if $\left|V_{\mathbb{R}}(g)\right|=10$, and if $g^{\prime}$ is another psd sextic with $V_{\mathbb{R}}(g) \subseteq V_{\mathbb{R}}\left(g^{\prime}\right)$, then $g, g^{\prime}$ have intersection number $\geq 10 \cdot 2^{2}=40$, implying $g^{\prime} \in \mathbb{R}_{+} g$ since $g$ is irreducible (see [9] Theorem 7.2 for this argument).

2. Since an absolutely irreducible plane sextic can have at most 10 singular points, the preceding remark shows that any exposed and non-sos psd sextic defines a rational curve. Therefore, and by Straszewicz's theorem, every extreme form in $P_{6} \backslash \Sigma_{6}$ defines a rational sextic. This argument was used in [1] to show that the Zariski closure in $\left|\mathcal{O}_{\mathbb{P}^{2}}(6)\right|$ of the extreme curves in $P_{6} \backslash \Sigma_{6}$ is contained in the Severi variety $S_{6,0}$ of plane rational sextics. In fact, the authors proved (loc. cit. Theorem 2) that the Zariski closure is equal to $S_{6,0}$, by observing that $S_{6,0}$ is irreducible of dimension 17 , and by producing a 17 -dimensional local family of extreme forms in $P_{3,6} \backslash \Sigma_{3,6}$, based on an analysis from [9. See also Remark 4.17 below.

3. Proposition 4.10 shows that for a generically chosen admissible set $S$, the extreme sextic $q_{S}$ has a tenth real zero, and is therefore exposed. Conversely, it follows from Corollary 3.8 that every exposed sextic $q$ is covered by construction 4.6. Actually this happens in ten different ways, since there are ten ways of choosing nine points out of ten.

Remark 4.17. Corollary 4.7 gives a very explicit argument for the fact that the set of extreme forms in $P_{3,6} \backslash \Sigma_{3,6}$ has (projective) dimension 17. Indeed, fixing a plane cubic curve $X$ with at most nodes as singularities, there is an 8-parameter family of admissible subsets $S$ of $X_{\text {reg }}(\mathbb{R})$. Adding the 9 parameters for choosing $X$, this together shows that the admissible sets form a 17-dimensional family. The map assigning to each admissible $S$ the extreme psd sextic $q_{S}$ is an explicit parametrization of the extreme sextics in $P_{6} \backslash \Sigma_{6}$ with at least 9 real zeros. Generically, this parametrization is a $10: 1$-map.

We finally discuss a geometric way to find the 10th zero of the extreme psd sextic $q_{S}$, for $S \subseteq \mathbb{P}^{2}(\mathbb{R})$ admissible. The following construction is extracted from Coble's remarkable paper 4 . We work over $\mathbb{C}$ in the next proposition.

Proposition 4.18. (Coble) Let $T \subseteq \mathbb{P}^{2}$ be a set with $|T|=8$, no 4 points on a line and no 7 on a conic. Choose cubic forms $f, f^{\prime}$ with $I_{3}(T)=\operatorname{span}\left(f, f^{\prime}\right)$ and a sextic form $g$ with $I_{6}(2 T)=\operatorname{span}\left(f^{2}, f f^{\prime}, f^{\prime 2}, g\right)$ (c.f. 3.2). Let $M$ be the 9th point of intersection of $f$ and $f^{\prime}$, and let $j=\operatorname{det} J\left(f, f^{\prime}, g\right)$, the Jacobian determinant.

(a) The curve $N_{T}=V(j)$ has degree 9 and depends only on $T$.

(b) Every point of $T$ is a triple point of $N_{T}$. 
(c) Assume that the cubic $X=V(f)$ is nonsingular. Then for $Q \in X, Q \notin$ $T \cup\{M\}$ we have

$$
\operatorname{dim} I_{6}(2 T+2 Q)>1 \Leftrightarrow 2 Q \sim 2 M \text { on } X \Leftrightarrow Q \in N_{T} .
$$

Moreover $M \notin N_{T}$ except when $M \in T$.

Note that for $X=V(f)$ nonsingular, (c) gives 27 intersection points of $X$ and $N_{T}$, at least in the generic case $M \notin T$, namely the three points $Q \neq M$ with $2 Q \sim 2 M$, together with the eight points in $T$ where the intersection index is 3 .

Proof. (a) follows from elementary properties of Jacobians, and (b) is readily seen in local coordinates. For (c) assume that $X=V(f)$ is nonsingular, and let $Q \in X$, $Q \notin T \cup\{M\}$. When $h$ is a sextic that is singular in $T \cup\{Q\}$ and not a multiple of $f^{2}$, then $\operatorname{gcd}(f, h)=1$ and $\operatorname{div}_{X}(h)=2 Q+2 \sum_{P \in T} P=2(Q-M)+\operatorname{div}_{X}\left(f^{\prime}\right)$, hence $2 Q \sim 2 M$ on $X$. On the other hand, $2 Q \sim 2 M$ implies the existence of a sextic $h \in I_{6}(2 T)$ with $\operatorname{gcd}(f, h)=1$ and $i_{Q}(f, h) \geq 2$, hence $J\left(f, f^{\prime}, h\right)$ is singular at $Q$. Conversely let $j(Q)=0$. Since $f^{\prime}(Q) \neq 0$, this means that $i_{Q}(f, g) \geq 2$, and arguing as in 4.1 we find a cubic $p$ such that $g+p f$ is singular in $T \cup\{Q\}$, showing $\operatorname{dim} I_{6}(2 T+2 Q)>1$. When $M \notin T$ then $f$ and $f^{\prime}$ meet transversally at $M$, so $j(M) \neq 0$.

Corollary 4.19. Let $S \subseteq \mathbb{P}^{2}(\mathbb{R})$ be an admissible set of nine points, let $P \in S$, and put $T=S \backslash\{P\}$. Then $P$ lies on the nonic curve $N_{T}$ (4.18).

Proof. There exists a sextic $g \in I_{6}(2 T) \backslash I_{3}(T)^{2}$ that is singular in $P$, for example the extreme psd sextic $q_{S}$ (4.8). Since $N_{T}$ has equation $j=\operatorname{det} J\left(f, f^{\prime}, g\right)=0$ where $I_{3}(T)=\operatorname{span}\left(f, f^{\prime}\right)$, it is clear that $j(P)=0$.

Remark 4.20. Let $S \subseteq \mathbb{P}^{2}(\mathbb{R})$ be an admissible set, and assume that the extreme psd sextic $q_{S}$ has a 10 th real zero $Q \notin S$. (Generically this is the case, see Proposition 4.10.) Corollary 4.19 offers a way of finding $Q$ (and therefore $q_{S}$ ), at least up to a finite choice. Indeed, $Q$ lies on the intersection $N_{S \backslash\{P\}} \cap N_{S \backslash\left\{P^{\prime}\right\}}$, for any choice of two points $P \neq P^{\prime}$ in $S$. See Example 5.5 for an illustration.

Remark 4.21. One may wonder about a characterization of the 10-point real zero sets of psd sextics, or in other words, of the real zero sets of exposed psd sextics. Such a characterization has been asked for in 3 . Let $U \subseteq \mathbb{P}^{2}(\mathbb{R})$ be a set with $|U|=10$. If a psd sextic $q$ with $V_{\mathbb{R}}(q)=U$ exists then every 9-point subset of $U$ is admissible. Conversely assume that $U \backslash\{P\}$ is admissible for every point $P \in U$. Does it follow that there exists a psd sextic $q$ with $V_{\mathbb{R}}(q)=U$ ? This seems likely, but we haven't been able to prove it. At any rate, our results permit to check, for any given $U$, whether there is a psd sextic with zeros in $U$ (and to find it in the positive case): Pick $P \in U$, decide whether $S=U \backslash\{P\}$ is admissible, and if yes, proceed to determine the extreme sextic $q_{S}$. If there exists a psd sextic through $U$ it has to be $q_{S}$, so a necessary and sufficient condition is that $q_{S}(P)=0$.

\section{EXAMPLES}

We are going to illustrate the general construction of admissible sets in the plane and their associated extreme psd sextics, as well as several phenomena discussed in the previous sections. For typographical simplicity we sometimes use homogeneous coordinates $(x, y, z)$ instead of $\left(x_{0}, x_{1}, x_{2}\right)$. 
5.1. For a first example we work in affine coordinates $(x, y)$. Let $X=V(f)$ be the elliptic curve with affine equation

$$
f(x, y, 1)=y^{2}-x^{3}-x^{2}-4 .
$$

Let + denote the abelian group law on $X(\mathbb{R})$ with neutral element the point at infinity. The group $X(\mathbb{R})$ has one connected component, with real 2-torsion point $Q=(-2,0)$. Let $P=(0,2)$, a non-torsion point on $X$. The nine points $S=$ $\{Q, \pm P, \pm 2 P, Q \pm P, Q \pm 2 P\}$ on $X$ add up to $Q$ in the group law of $X$. Therefore the set $S$ is admissible. Each of the four triples $(P, Q+P, Q-2 P),(-P, Q-$ $P, Q+2 P),(Q, 2 P, Q-2 P),(Q,-2 P, Q+2 P)$ lies on a line, and the six points $( \pm P, \pm 2 P, Q \pm P)$ lie on a conic. The product is a sextic that is singular in the points of $S$, namely $q=l_{1} l_{2} l_{3} l_{4} p$ where $l_{1}=3 x+y-2, l_{2}=3 x-y-2, l_{3}=2 x+y+4, l_{4}=$ $2 x-y+4$ and $p=y^{2}-2 x^{2}-2 x-4$. By construction, $q$ has constant (nonnegative) sign on $X(\mathbb{R})$. The minimal value $t \in \mathbb{R}$ for which the sextic $q_{t}=q+t f^{2}$ is psd gives the extreme sextic $q_{S}=q_{t}$ in $P_{6}(S) \backslash \Sigma_{6}(S)$. One finds that $q_{S}$ is exposed with 10 th real zero at $(\alpha, 0)$, where $\alpha \approx 4.25925$ is the real root of $5 x^{3}-15 x^{2}-24 x-12$. The corresponding value for $t$ is $t=\frac{1}{49}\left(165 \alpha^{2}+60 \alpha+1156\right) \approx 89.89509$.

5.2. For a second example let $X=V\left(x_{0} x_{1} x_{2}\right)$, union of three lines in general position. Write $P_{0}(a)=(0: a: 1), P_{1}(a)=(1: 0: a)$ and $P_{2}(a)=(a: 1: 0)$ for $a \in \mathbb{R}^{*}$. The Picard group of $X$ is an extension $1 \rightarrow \mathbb{R}^{*} \rightarrow \operatorname{Pic}(X) \rightarrow \mathbb{Z}^{3} \rightarrow 0$. Given $a_{i}, a_{i}^{\prime} \in \mathbb{R}^{*}(i=0,1,2)$ we have

$$
\sum_{i=0}^{2} P_{i}\left(a_{i}\right) \sim \sum_{i=0}^{2} P_{i}\left(a_{i}^{\prime}\right) \text { in } \operatorname{Div}(X) \quad \Leftrightarrow \quad a_{0} a_{1} a_{2}=a_{0}^{\prime} a_{1}^{\prime} a_{2}^{\prime} .
$$

So if $a_{0}, a_{1}, a_{2} \in \mathbb{R}^{*}$, the three points $P_{i}\left(a_{i}\right)(i=0,1,2)$ lie on a line if and only if $a_{0} a_{1} a_{2}=-1$. Therefore, $S \subseteq X_{\text {reg }}(\mathbb{R})$ is admissible if and only if there exist $a_{i}, b_{i}, c_{i} \in \mathbb{R}^{*}$ with $\left|\left\{a_{i}, b_{i}, c_{i}\right\}\right|=3(i=0,1,2)$ such that

$$
S=\left\{P_{i}\left(a_{i}\right), P_{i}\left(b_{i}\right), P_{i}\left(c_{i}\right): i=0,1,2\right\}
$$

and

$$
a_{0} a_{1} a_{2} \cdot b_{0} b_{1} b_{2} \cdot c_{0} c_{1} c_{2}=1 .
$$

For an explicit example let

$$
S=\left\{P_{i}( \pm 1): i=0,1,2\right\} \cup\left\{P_{0}\left(\frac{3}{2}\right), P_{1}\left(\frac{1}{3}\right), P_{2}(-2)\right\} .
$$

A sextic that is singular in the points of $S$ is

$$
q=\left(x_{0}+x_{1}+x_{2}\right)\left(x_{0}+x_{1}-x_{2}\right)\left(x_{0}-x_{1}+x_{2}\right)\left(x_{0}-x_{1}-x_{2}\right)\left(x_{0}+2 x_{1}-3 x_{2}\right)^{2}
$$

The extreme psd sextic associated to $S$ is $q_{S}=q+t\left(x_{0} x_{1} x_{2}\right)^{2}$ where $t \approx 114.68148$. Again, $q_{S}$ is exposed with 10 th real zero $(\alpha: \beta: 1)$ where $\alpha \approx-0.64185$ is the smallest real root of $27 x^{3}-111 x^{2}-59 x+15$ and $\beta=\frac{9}{16}\left(1-\alpha^{2}\right)+2 \alpha \approx-0.95295$. (The above number $t$ is also a rational expression in $\alpha$.)

5.3. This is an example of an admissible set $S$ for which the extreme psd sextic $q_{S}$ has only the 9 real zeros in $S$. Let $S$ consist of the 9 points

$$
(0: 1: \pm 1),(1: 0: \pm 1),(1: \pm 1: 0),(3: 0: 2),(3: 2: 0),(5: 4: 4) \text {. }
$$


One can show that the set $S$ is admissible, the unique cubic $X=V(f)$ through $S$ being given by

$$
f=8(x+y+z)\left(2 x^{2}+3 y^{2}+3 z^{2}-5 x y-5 x z-6 y z\right)+195 x y z .
$$

$X$ is an elliptic curve for which $X(\mathbb{R})$ has two connected components. Let $l=$ $2 x-3 y-3 z$, and let $l_{1}, l_{2}, l_{3}, l_{4}$ be the four linear forms $x \pm y \pm z$. The extreme psd sextic with zeros in $S$ is

$$
q_{S}=2496 \cdot l_{1} l_{2} l_{3} l_{4} l^{2}+(888 f+273 x y z)^{2}
$$

The sextic $q_{S}$ has no real zero beyond $S$, rather the point $(0: 1:-1) \in S$ is an $A_{3}$-singularity of $q_{S}$.

5.4. The Robinson sextic [10] is the unique psd sextic through the 10 points that arise from permuting the coordinates of $(1: 1: \pm 1)$ and $(0: 1: \pm 1)$. It has symmetric equation

$$
R=\left(x^{6}+y^{6}+z^{6}\right)-\left(x^{4} y^{2}+x^{4} z^{2}+y^{4} x^{2}+y^{4} z^{2}+z^{4} x^{2}+z^{4} y^{2}\right)+3 x^{2} y^{2} z^{2} .
$$

Let $T$ consist of the 8 points $( \pm 1: \pm 1: 1),( \pm 1: 0: 1),(0: \pm 1: 1)$. Then $I_{3}(T)$ is the pencil generated by $f=x^{3}-x z^{2}$ and $f^{\prime}=y^{3}-y z^{2}$. Therefore $I_{6}(2 T)$ is spanned by $f^{2}, f f^{\prime}, f^{\prime 2}$ and $R$. The nonic $N_{T}=\operatorname{det} J\left(f, f^{\prime}, R\right)$ has equation

$$
j=z\left(z^{2}-x^{2}\right)\left(z^{2}-y^{2}\right)\left(x^{2}+x y+y^{2}-z^{2}\right)\left(x^{2}-x y+y^{2}-z^{2}\right) .
$$

Except for the line $z=0$, every irreducible component of $N_{T}$ is contained in some cubic from the pencil $I_{3}(T)$. Therefore, every admissible set $S=T \cup\{P\}$ has its 9th point $P$ on the line $z=0$.

Conversely fix $u \in \mathbb{R} \cup\{\infty\}$, let $P_{u}=(1: u: 0)$ (with $P_{\infty}=(0: 1: 0)$ ) and put $S_{u}=T \cup\left\{P_{u}\right\}$. Then the set $S_{u}$ is admissible for all $u \notin\{0, \infty\}$. Indeed, there is a unique cubic through $S_{u}$, namely $X_{u}=V\left(f_{u}\right)$ with $f_{u}=u^{3} f-f^{\prime}$. And the definiteness condition is satisfied since $X_{u}(\mathbb{R})$ is connected (resp. $X_{u}$ is a union of a line and a conic with two real intersection points for $u= \pm 1$ ). For $u=0, \infty$ the set $S_{u}$ fails to be admissible since $P_{u}$ is a triple point of $X_{u}$.

Let $u \neq 0, \infty$. To find the extreme sextic $q_{u}:=q_{S_{u}}$, and possibly its 10 th real zero $Q_{u}$, we may use the Coble nonic as in 4.20. So we may pick $P^{\prime}=(1: 1:-1)$ (or any other point in $T$ ), put $T_{u}^{\prime}=S_{u} \backslash\left\{P^{\prime}\right\}$, compute the nonic $N_{T_{u}^{\prime}}$ and intersect it with $N_{T}$. One finds that $N_{T_{u}^{\prime}}$ intersects the line $z=0$ of $N_{T}$ in the four real points $(1: 0: 0),(0: 1: 0),(u: 1: 0)$ and $(1:-u: 0)$, besides $P_{u}$. The sextics in $I_{6}\left(2 S_{u}\right)$ through the first three points are indefinite. Therefore we must have $Q_{u}=(1:-u: 0)$. The corresponding sextic is

$q_{u}=u^{6}\left(u^{2}+2\right)\left(x^{3}-x z^{2}\right)^{2}-3 u^{4}\left(x^{2}-z^{2}\right)\left(y^{2}-z^{2}\right)\left(x^{2}+y^{2}-z^{2}\right)+\left(2 u^{2}+1\right)\left(y^{3}-y z^{2}\right)^{2}$ which must be the exposed psd sextic with ten zeros in $T \cup\{(1: \pm u: 0)\}$. For $u= \pm 1$ we get $q_{u}=3 R$.

Note that the example of $T$ is special since there exist lines containing three points of $T$ (and conics containing six points of $T$ ). These lines and conics are necessarily irreducible components of the nonic $N_{T}$.

5.5. For every real number $u$, the symmetric sextic

$$
q_{u}=a \sum x_{i}^{4} x_{j}^{2}+b \sum x_{i}^{4} x_{j} x_{k}+c \sum x_{i}^{3} x_{j}^{3}+d \sum x_{i}^{3} x_{j}^{2} x_{k}+e\left(x_{0} x_{1} x_{2}\right)^{2}
$$

with

$$
a=u^{2}, b=-2\left(u^{2}-2\right), c=2 u^{2}, d=-2\left(u^{2}+u+2\right), e=6\left(u^{2}+4 u+2\right)
$$


is singular in the points that arise from

$$
(1: 0: 0),(1:-1: 0),(1: 1: u),(1: 1: 1)
$$

by permuting the coordinates. For $u \neq 1$ these are 10 points. The following hold:

(a) $q_{u}$ is psd and not sos for $u<-2$ or $u>1$,

(b) $q_{u}$ is a sum of two squares for $u=-2$, and a square for $u=1$,

(c) $q_{u}$ is indefinite for $-2<u<1$.

Here is a way to see this. Let $S_{u}$ be the set of points obtained by permuting the coordinates of $(1: 0: 0),(1:-1: 0)$ and $(1: 1: u)\left(\right.$ so $\left|S_{u}\right|=9$ for $\left.u \neq 1\right)$. We need to find the values of $u$ for which $S_{u}$ is admissible. For $u=1$ we have $\left|S_{u}\right|=7$, for $u=-2$ the line $x_{0}+x_{1}+x_{2}=0$ contains six points of $S_{u}$. For any $u$ the symmetric cubic $X_{u}=V\left(f_{u}\right)$ with

$$
f_{u}=2\left(u^{2}+u+1\right) x_{0} x_{1} x_{2}-u \sum x_{i}^{2} x_{j}
$$

passes through $S_{u}$, and for $u \notin\{1,-2\}$ it is the only cubic through $S_{u}$. For the singular values $u \in\left\{-2,-\frac{1}{2}\right\}, X_{u}$ is the union of a line and a conic without real intersection point, and for $u \in\{-1,0\}, X_{u}$ is a triangle whose singularities lie in $S_{u}$. Therefore $S_{u}$ is not admissible for $u \in\left\{-2,-1,-\frac{1}{2}, 0,1\right\}$. For all $u \notin$ $\left\{-2,-1,-\frac{1}{2}, 0,1\right\}$, the cubic $X_{u}$ is nonsingular, and $X(\mathbb{R})$ has two connected components. For any of these values $u$, the divisor class $\left[\mathcal{O}_{X_{u}}(3)\right]-\sum_{P \in S_{u}}[P]$ on $X_{u}$ is a nonzero 2-torsion element in $\operatorname{Pic}\left(X_{u}\right)$. To find the values $u$ for which this class is definite (and hence $S_{u}$ is admissible), we follow the geometric approach in 3.11. Let $P=(1: 0: 0) \in S_{u}$ and put $T_{u}=S_{u} \backslash\{P\}$. The 9 th intersection point of the pencil $I_{3}\left(T_{u}\right)$ is $M_{u}=(u: 1: 1)$ (which happens to lie in $\left.T_{u}\right)$. The tangents to $X_{u}$ at $P$ and $M_{u}$ are $t_{P}=x_{1}+x_{2}$ and $t_{M_{u}}=x_{0}-u x_{1}-u x_{2}$, so they intersect at $(0: 1:-1) \in T_{u}$. A geometric sketch shows that the product $t_{M_{u}} \cdot t_{P}$ is indefinite on $X_{u}(\mathbb{R})$ if and only $-2<u<1$.

The discussion shows that for $-2<u<1$, the only psd sextic through $S_{u}$ is $f_{u}^{2}$. On the other hand, there exist psd non-sos sextics through $S_{u}$ when $u<-2$ or $u>1$, and the unique extreme one is $q_{u}$.

\section{REFERENCES}

[1] G. Blekherman, J. Hauenstein, J. C. Ottem, K. Ranestad, B. Sturmfels: Algebraic boundaries of Hilbert's SOS cones. Compositio Math. 148, 1717-1735 (2012).

[2] S. Bosch, W. Lütkebohmert, M. Raynaud: Néron Models. Erg. Math. Grenzgeb. (3) 21, Springer, Berlin, 1990.

[3] M.D. Choi, T. Y. Lam, B. Reznick: Real zeros of positive semidefinite forms. I. Math. Z. 171, 1-26 (1980).

[4] A. B. Coble: The ten nodes of the rational sextic and of the Cayley symmetroid. Am. J. Math. 41, 243-265 (1919).

[5] R. Hartshorne: Algebraic Geometry. Grad. Texts Math. 52, Springer, New York, 1977.

[6] D. Hilbert: Über die Darstellung definiter Formen als Summe von Formenquadraten. Math. Ann. 32, 342-350 (1888).

[7] D. Hilbert: Über ternäre definite Formen. Acta math. 17, 169-197 (1893).

[8] B. Reznick: Some concrete aspects of Hilbert's 17th problem. In: Real Algebraic Geometry and Ordered Structures (Ch. Delzell, J. Madden eds.), Contemp. Math. 253, AMS, 2000, pp. 251-272.

[9] B. Reznick: On Hilbert's construction of positive polynomials. Preprint, arxiv:0707.2156.

[10] R. M. Robinson: Some definite polynomials which are not sums of squares of real polynomials. (Russian) In: Izdat. Nauka Sibirsk. Otdel., Novosibirsk, 1973 (Selected questions of algebra and logic, collection dedicated to the memory of A. I. Mal'cev)), pp. 264-282. 
[11] R. T. Rockafellar: Convex Analysis. Princeton University Press, Princeton NJ, 1970.

[12] C. Scheiderer: Sums of squares of regular functions of real algebraic varieties. Trans. Am. Math. Soc. 352, 1039-1069 (1999).

Fachbereich Mathematik und Statistik, Universität Konstanz, Germany 